ARTÍCULO ORIGINAL

\title{
Prevalencia de helmintos intestinales en caninos del departamento del Quindío
}

\author{
María Isabel Giraldo, Nora Lizeth García, Jhon Carlos Castaño \\ Grupo de Inmunología Molecular, Centro de Investigaciones Biomédicas, Universidad del Quindío, \\ Armenia, Colombia.
}

Introducción. Los helmintos intestinales son agentes patógenos que afectan animales domésticos y que a través de ellos pueden infectar humanos.

Objetivo. El objetivo del trabajo fue determinar la prevalencia de helmintos intestinales en perros con dueño del departamento del Quindío.

Materiales y métodos. Estudio descriptivo prospectivo. Se aplicó una encuesta epidemiológica a los propietarios de los perros. Se recolectaron muestras de heces de los caninos registrados en la jornada de vacunación antirrábica del 2003 en el departamento del Quindío. Las muestras de materia fecal frescas fueron analizadas utilizando la técnica de diagnóstico de Ritchie.

Resultados. Se analizaron 324 muestras de heces caninas; el 67,6\% de los perros eran de razas puras y el 32,4\% razas mestizas. Se encontró una prevalencia del 22,2\%; Ancylostoma caninum fue el parásito más frecuente, 13,9\%. También se observó Trichuris vulpis, 4,3\%; Toxocara canis, 2,5\%, y Strongyloides stercoralis, 4,0\%. El 2,46\% de las mascotas se encontraron multiparasitadas.

Conclusión. La frecuencia de helmintos intestinales en el departamento del Quindío fue de $22,2 \%$ y la presencia de estos parásitos coincide con factores como la edad y la permanencia del canino en la calle, entre otros. Por esta razón, es necesario establecer programas de vigilancia y prevención en la población humana y canina.

Palabras clave: prevalencia, enfermedades de los perros, helmintos, Ancylostoma, Toxocara canis, Strongyloides stercoralis.

\section{Prevalence of intestinal helminths in dogs from Quindío Province}

Background. Intestinal helminths are pathogens for domestic animals and provide a source of potential infection for humans.

Objectives. The prevalence of intestinal helminths in domestic dogs was determined in a province-wide survey in Quindío Province, Colombia.

Materials and methods. The sample size was calculated based upon the data of the 2003 antirabies vaccination program in Quindio. Information in the form of an epidemiological questionnaire was provided by dog owners. Fecal samples from dogs were analyzed by Ritchie's concentration method.

Results. Of 324 samples, $67.6 \%$ were from purebred dog races and $32.4 \%$ were mongrels. A $22.2 \%$ prevalence for intestinal helminthes was found. Ancylostoma caninum was the most prevalent parasite (13.9\%), followed by Trichuris vulpis (4.3\%), Toxocara canis (2.5\%), and Strongyloides stercoralis (4.0\%). Multiparasite infestations were observed in $2.46 \%$ of the dogs. Conclusion. Presence of parasites was strongly correlated with age and degree of association with the open streets. Control programs are recommended for helminth surveillance in human and canine populations.

Keywords: prevalence, dog diseases, helminths, Ancylostoma, Toxocara canis, Strongyloides stercoralis. 
Los helmintos intestinales son agentes patógenos importantes que afectan al hombre y animales de compañía; muchos de estos parásitos se consideran de importancia zoonótica, pues existe una mayor probabilidad de contagio en los niños, dado que frecuentan sitios públicos de recreación y esparcimiento como plazas y parques donde perros con estado sanitario desconocido defecan (1-3).

En las últimas décadas, la sociedad colombiana ha experimentado cambios que han modificado hábitos y conductas, entre las que se presenta una tendencia creciente a la tenencia de mascotas, y los caninos y los felinos son las especies animales de mayor preponderancia (1-5). La mayoría de las mascotas son tratadas como miembros de la familia, compartiendo con ellas la habitación y el mismo espacio para dormir, sin considerar el posible riesgo de transmisión de enfermedades zoonóticas (1-5); además, pueden ser portadoras de enfermedades virales, bacterianas y parasitarias que afectan, principalmente, a los niños.

Entre los helmintos intestinales que afectan a los caninos se encuentran: Ancylostoma caninum, Trichuris vulpis, Strongyloides stercoralis, Dipylidium caninum y Toxocara canis, entre otras; éstos ocasionan deterioro de la salud animal debido a que afectan el bienestar y la vitalidad del hospedero y, en casos extremos, ocasionan la muerte. Los caninos afectados experimentan anorexia y excreción de parásitos adultos en el vómito o las heces. En las infecciones masivas los perros presentan abdomen abultado, mala condición del pelaje, diarrea y retardo en el desarrollo. El diagnóstico de helmintiasis se puede realizar por observación microscópica de concentrados de huevos o larvas, a partir de muestras de materia fecal o la visualización macroscópica de los adultos (6).

Uno de los factores de riesgo es, justamente, la poca información que se tiene sobre las parasitosis

\footnotetext{
Correspondencia:

Jhon Carlos Castaño, Carrera 15 calle 12 norte, Armenia, Quindío, Colombia.

Telefax: +(57 67) 460168

gymol@uniquindio.edu.co
}

Recibido: 14/02/05; aceptado: 09/06/05 caninas en la región y sobre el ciclo de vida de los parásitos, al igual que su relación con los hospederos, con respecto a la dinámica de población, concretamente la cantidad de caninos, niños y adultos que circulan, la carga parasitaria, y la cantidad de agentes etiológicos (heces contaminadas con huevos o larvas de helmintos) (7).

En nuestro país se han desarrollado muy pocos estudios sobre la prevalencia de parásitos intestinales en caninos. En el departamento del Huila, Penagos et al. realizaron en 2004 un trabajo en el que se encontraron prevalencias representativas de $A$. caninum con $86,8 \%$, de $T$. canis con $13,6 \%$ y de $T$. vulpis con $3 \%$, relacionados con el $37,4 \%$ de las muestras positivas para huevos, larvas o quistes de parásitos (8). En Bogotá se realizó un trabajo similar y se estimó la prevalencia de los helmintos gastrointestinales en caninos errantes recolectados por el Centro de Zoonosis; los parásitos hallados con mayor frecuencia fueron los Ancylostómidos que variaron entre $50 \%$ y $84,8 \% ;$ T. canis mostró su mayor prevalencia en los cachorros de 0 a 6 meses, con 23,86\% (3); en el Quindío, hasta el momento, no se han registrado estudios que permitan identificar el estado del parasitismo intestinal en la población canina del departamento $y$, por lo tanto, nos propusimos determinar la prevalencia de helmintos intestinales en caninos del departamento del Quindío.

\section{Materiales y métodos}

Se realizó un estudio prospectivo de tipo descriptivo en la población canina con dueño que fue registrada en la jornada de vacunación antirrábica del 2003 en departamento del Quindío. Mediante un muestreo probabilístico aleatorio estratificado con un error del $5 \%$ y un nivel de confianza del $95 \%$, con un estimativo de la prevalencia del $45 \%$, se tomaron 324 muestras de heces caninas durante el segundo semestre del 2003 y todo el 2004, en todos los municipios del departamento del Quindío.

\section{Procedimientos y toma de muestras}

A cada persona responsable o propietario de mascota se le realizó una encuesta epidemiológica 
en la que se consignaron datos demográficos, hábitos del canino, contacto con otros caninos, alimentación, control veterinario y sintomatología; se tuvo en cuenta la condición corporal de cada canino si era buena, regular o mala, determinada por medio de la observación y la palpación. Las categorías planteadas se adaptaron del manual de la Hill's Pet Nutrition (9).

Se tomaron muestras de materia fecal a cada animal seleccionado; las heces debían ser frescas sin contaminación con tierra, sustancias extrañas o heces de otros animales. La recolección se hizo en horas de las mañana mediante visita domiciliaria en cada uno de los municipios del departamento del Quindío. Los recipientes plásticos se llenaron completamente de materia fecal hasta el borde, con el fin de eliminar el aire $y$, así, disminuir la velocidad de desarrollo y eclosión de los huevos. Posteriormente, las muestras fueron transportadas al laboratorio en nevera de icopor a $4^{\circ} \mathrm{C}$ para, luego, ser analizadas rápidamente. Si las muestras no se observadas al microscopio el mismo día de la recolección, se preservaban en formol al $10 \%$ hasta su procesamiento utilizando la técnica modificada de Ritchie; dichas modificaciones consisten en la disminución del número de centrifugaciones y la adición de la muestra directamente en formol al $10 \%(10)$.

La identificación de los parásitos se basó en sus características morfológicas y en las mediciones biométricas de sus huevos en el microscopio. La determinación de la prevalencia se basó en los resultados obtenidos por el método modificado de Ritchie. Los datos que se obtuvieron en la aplicación de la encuesta y en el procesamiento de las muestras se organizaron en tablas y, luego, se relacionaron las variables frente a los resultados obtenidos.

\section{Resultados}

\section{Aspectos epidemiológicos}

Raza. El análisis de los datos consignados en las encuestas epidemiológicas mostró que la población canina estudiada en nuestro departamento por razas puras con $67,6 \%$ y las razas mestizas en $32,4 \%$.
Edad. Se encontraron 87 animales de 1 mes a 1 año; 124, de 1 a 4 años, y 113 mayores de 4 años.

Condición corporal. La condición corporal observada en los caninos fue mala (14 muestras), regular (51 muestras) y buena (259 muestras); la presencia de parásitos fue de 7 perros con condición mala, 19 en perros con condición regular y 46 en perros con condición buena.

\section{Hábitos alimenticios}

Se encontró que el $40,7 \%$ de la población canina estudiada era alimentada con concentrado, el $15,1 \%$ con comida casera y al $44,1 \%$ se le suministraban los dos tipos de alimentación. Con relación al suministro de agua, el $76,2 \%$ de los propietarios les suministraba agua cruda; el $18,5 \%$, agua hervida; $4,0 \%$, de ambas, y el 1,3\% les proporcionaba otro tipo de bebidas como leche o agua de panela.

\section{Lugar de eliminación de excretas}

El 34\% de los caninos disponen las heces fuera de la casa, el $50,3 \%$ dentro de la casa y el $16 \%$ utilizan ambos lugares.

\section{Sintomatología}

De acuerdo con los síntomas que presentaron los animales sólo se tuvo en cuenta la presencia de diarrea y la relación con $T$. canis, ya que se obtuvo una relación estadísticamente significativa $(\mathrm{p}<0,005)$.

\section{Presencia de parásitos}

Se encontró que $A$. caninum fue el parásito más prevalente $(13,9 \%)$, seguido por T. vulpis $(4,3 \%)$, S. stercoralis $(4,0 \%)$ y $T$. canis $(2,5 \%)$. Además, se evidenció biparasitismo en 8 individuos, así: A. caninum $+T$. vulpis en 6 caninos $(1,8 \%)$ y $S$. stercoralis $+T$. vulpis en 2 caninos $(0,6 \%)$.

De los 324 caninos estudiados, el 22,2\% resultó parasitado con alguna especie de helminto adulto, evidenciado por la presencia de huevos en las heces de los animales procesadas mediante la técnica de concentración de Ritchie.

El porcentaje de animales positivos fue similar para machos $(23,8 \%)$ y hembras $(20,6 \%)$, respectivamente, y la frecuencia de animales 
infectados se incrementa según la edad (cuadro 1). Además, el rango de edad más frecuente estuvo entre 1 y 4 años, seguido por los mayores de 4 años y los menores de 1 año; estos últimos fueron los de mayor frecuencia parasitaria (cuadro 1).

La condición corporal de los caninos estudiados fue: mala en $4,3 \%$, grupo en el que el porcentaje de parasitismo fue del $50 \%$, mientras las mascotas con buena condición corporal representaron el $79,9 \%$, y de éstos sólo el $17,7 \%$ presentó infección por helmintos intestinales. Al realizar pruebas estadísticas para relacionar las variables de condición corporal y edad no se encontraron diferencias estadísticamente significativas.

\section{Discusión}

A nivel mundial existe el reporte de prevalencias de helmintos intestinales en caninos entre $4 \%$ y $78,0 \%$, determinadas por medio del análisis en materia fecal y en inspección post mortem (1119). En Colombia se han reportado prevalencias entre el $37,4 \%$ y el $76 \%$ de positividad a huevos, larvas y quistes de parásitos en heces de los caninos examinados $(3,8)$. No existen reportes previos ni para Quindío ni para la zona central del país.

En el presente estudio se encontró una prevalencia de $22,2 \%$, la cual se encuentra dentro de las prevalencias reportadas en el mundo. Sin embargo, es inferior a lo reportado a nivel nacional, $76 \%$ en Bogotá en 2003 y 37,4\% en Huila en 2004 $(3,8)$. Estas diferencias pueden deberse a

Cuadro1. Distribución por frecuencia y porcentual de la edad de la muestra de 324 caninos en el departamento del Quindío.

\begin{tabular}{lcccc}
\hline Variable & $\begin{array}{c}\text { No. de } \\
\text { muestras de } \\
\text { heces }\end{array}$ & (\%) & $\begin{array}{c}\text { No. de animales } \\
\text { positivos para } \\
\text { helmintos }\end{array}$ & (\%) \\
\hline recolectadas & & 29 & 33,3 \\
Edad & 87 & 26,8 & 29 & \\
$\begin{array}{l}\text { De } 1 \text { mes } \\
\text { a } 1 \text { año }\end{array}$ & 124 & 38,2 & 27 & 21,7 \\
$\begin{array}{l}\text { De } 1 \text { año } \\
\text { a } 4 \text { años } \\
\text { Mayor de } \\
4 \text { años }\end{array}$ & 113 & 34,8 & 16 & 14,1 \\
\hline
\end{tabular}

variaciones ambientales, principalmente de temperatura y humedad dado que los estudios reportados se han realizado en zonas más secas, con diferencias en la altitud y la temperatura (Bogotá: 2.640 metros sobre nivel del mar (msnm)), $14^{\circ} \mathrm{C}$ y humedad relativa de $72 \%$; Huila: $442 \mathrm{msnm}, 28^{\circ} \mathrm{C}$ y $80 \%$ de humedad relativa) frente a temperaturas que varían entre $12^{\circ} \mathrm{C}$ y $21^{\circ} \mathrm{C}$, altitud entre 1.200-1.923 msnm y una humedad relativa de $85 \%$ en el departamento del Quindío.

Anteriormente se había reportado que las parasitosis son más frecuentes en las áreas tropicales y subtropicales (20). Estas condiciones propician el desarrollo y la persistencia parasitaria, debido a que los helmintos necesitan ambientes cálidos y húmedos que favorecen su supervivencia (21). La temperatura media para la evolución de la fase externa de este tipo de parasitismo es de $25^{\circ} \mathrm{C}$ y requiere un mínimo de $50 \mathrm{~mm}$ de lluvia por mes y una humedad relativa del $60 \%$ al $70 \%$. Las condiciones extremas impiden la eclosión del huevo y originan la muerte de la larva infectante en la postura.

En general, en la estación invernal, el frío retarda la eclosión de los huevos e inmoviliza a algunos estados larvarios que, al permanecer en estado de latencia, necesitan condiciones propicias para completar el ciclo. En el verano se acelera la eclosión de los huevos, aunque las temperaturas extremas producen su desecación y la destrucción de ciertas formas larvarias (22).

De igual manera, estas condiciones climáticas son favorables para la supervivencia de ratones, cucarachas, lagartijas, etc. que actúan como hospederos intermediarios en algunas parasitosis, lo cual incrementa el riesgo de infección en los animales susceptibles y la prevalencia parasitaria (23).

De acuerdo con la edad, el grupo de animales con mayor prevalencia de infección por helmintos intestinales correspondió a los menores de un año con $33,3 \%$ y los que presentaron menor prevalencia fueron los de 4 años en adelante con $14,1 \%$; un factor que puede influir en el alto porcentaje de parasitismo en cachorros es el hecho de que la inmunidad comienza a manifestarse a partir de la quinta semana de edad como ocurre 
en el caso de $T$. canis (24) y, también, las vías de transmisión parasitaria transplacentaria y transmamaria por lo que el cachorro puede infectarse desde antes de nacer o desde el mismo momento en que empieza a alimentarse de la madre (25).

En los grupos de edad, $A$. caninum y $T$. canis mostraron su mayor prevalencia en los perros menores de un año con $37,7 \%$ y $87,5 \%$, respectivamente, mientras que $T$. vulpis y $S$. stercoralis tuvieron prevalencias menores en este rango de edad, contrario a los perros que se encontraban entre 1 y 4 años los cuales tuvieron las mayores prevalencias para estos parásitos con $71,4 \%$ para $T$. vulpis y $53,8 \%$ para S. stercoralis.

$T$. canis presentó el mayor porcentaje en cachorros de 0 a 1 año debido, tal vez, a que el ciclo biológico del parásito presenta diferentes formas de infección; los cachorros y los neonatos entre 2 semanas y 2 meses de vida se afectan más por la vía transplacentaria y lactogénica (25).

El alto porcentaje de positividad para Ancylostómidos en caninos en todos los rangos de edad puede deberse a la ruta de transmisión percutánea, ya que los animales de la muestra salían a la calle donde eliminaban sus heces y daban paseos con sus amos, lo cual permite que estén en frecuente contacto con el suelo contaminado.

La presencia simultánea de parásitos adultos y de diarrea concuerda con lo reportado por Cordero del Campillo (23), en el sentido de que en las fases agudas de las infecciones producidas por los helmintos intestinales se producen episodios diarreicos, lo que le da importancia a este tipo de infecciones ya que muchas veces puede llevar a la muerte.

En el presente estudio no se encontraron diferencias con las especies reportadas en el mundo. Al analizar la prevalencia por especie de helmintos intestinales, los resultados que reporta este estudio para $A$. caninum son inferiores a los reportados en Colombia $(3,8)$ lo cual es atribuible al número de muestras, la edad y las condiciones ecológicas disímiles. A nivel mundial, estos resultados se encuentran dentro de los valores obtenidos por otros autores $(3,8,12-15,17,24$, $27,30,31)$.

Los resultados obtenidos para $T$. vulpis en Huila son similares a los obtenidos en este estudio; a nivel mundial mostró el mismo comportamiento a diferencia de los datos reportados por Fok et al. en Hungría (28), quien encontró $21,85 \%$ de prevalencia.

La prevalencia hallada por este estudio para S. stercoralis es semejante a la reportada en Huila (8) aunque en el mundo sólo se ha reportado en Nigeria donde la prevalencia fue de $18,8 \%(13,14)$.

$T$. canis fue el parásito de menor prevalencia en este estudio aunque en Colombia y a nivel mundial se ha mostrado ser superior en los hallazgos realizados; esto se explica, en parte, debido a que en este estudio los perros la muestra de problación de cachorros fue la menor $(3,8,12-15$, 17,27-29,31-34).

En el presente estudio no se consideró la frecuencia de cada especie parasitaria en relación con la condición corporal pero es evidente que estas infecciones producen trastornos gastrointestinales (irritación de la mucosa, mala absorción, diarrea, vómito y anemia, en el caso de A. caninum) que, unidos al tiempo de infección, inducen un descenso del consumo de alimento, lo que genera la movilización de las reservas del hospedero, y produce debilidad y disminución de la condición corporal $(16,27)$.

A. caninum, S. stercoralis y $T$. canis, parásitos encontrados en el presente estudio, son de relevancia médica dada su condición de entidades zoonóticas; por lo tanto, es necesario instaurar como medida de control la desparasitación periódica de las mascotas y educar a los propietarios en cuanto a la correcta eliminación de excretas debido a que ésta es la principal vía diseminación de parásitos en parques, áreas comunales de conjuntos cerrados y en el hogar, y evitar que los niños jueguen con los excrementos de animales domésticos.

\section{Agradecimientos}

Los autores agradecen al Centro de Investigaciones Biomédicas de la Universidad del 
Quindío, al Centro de Zoonosis de Armenia, al Instituto Seccional de Salud del Quindío, a la Secretaria de Salud de Armenia y al Laboratorio de Parasitología Veterinaria de la Universidad Nacional de Colombia por toda la colaboración prestada.

\section{Conflicto de interés}

Declaramos que no existen conflictos de interés con la institución donde laboramos, ni existen relaciones financieras o personales con otras personas $\mathrm{u}$ organizaciones que pueden influir $\mathrm{o}$ sesgar la forma de presentar los resultados.

\section{Financiación}

El presente trabajo fue financiado por la Universidad del Quindío.

\section{Referencias}

1. Taranto NJ, Passamonte L, Marinconz R, De Marzi MC, Cajal SP, Malchiodi EL. Parasitosis zoonóticas transmitidas por perros en el Chaco salteño. Medicina (Buenos Aires) 2000;60:217-20.

2. Minvielle MC, Pezzani BC, Basualdo Farjat JA. Frecuencia de hallazgo de huevos de helmintos en materia fecal canina recolectada en lugares públicos de la ciudad de La Plata, Argentina. Bol Chil Parasitol 1993;48:63-5.

3. Cabrera PA, Ordóñez OE, Cortés JA, Rodríguez JM, Villamil LC. Determinación de parásitos zoonóticos (helmintos y protozoarios) en caninos del Centro de Zoonosis de Bogotá, D.,C. Biomédica 2003;23(Sup. 1):153.

4. Codeisa. Comportamiento de la población canina del Distrito Capital analizando tasa de fecundidad, natalidad y mortalidad y la relación hombre-animal. Bogotá, D.C.: Secretaría Distrital de Salud de Bogotá, D.C.; 1999. p.77.

5. Herrera A. Prevalencia, intensidad y factores asociados a la presencia de nemátodos y céstodos intestinales, en gatos callejeros de la ciudad de Mérida, Yucatán. (tesis) México, D.F.: Universidad Autónoma de México; 2002.

6. Coffin DI. Laboratorio clínico en medicina veterinaria. $3^{a}$ ed. México, D. F: La Prensa Médica Mexicana, S.A.; 1986. p.847.

7. Fabijan Co. Indicadores de riesgo demográfico de transmisión de helmintiasis caninas en plazas de la ciudad de Buenos Aires. Cátedra de Parasitología, Facultad de Ciencias Exactas y Naturales, Universidad de Buenos Aires. 1997. Disponible en: http/ www.geocities,com/Athens/Atlantis/4003/textos/ parásito.
8. Penagos J, Ardila A, Fernández J, Vargas J, Lozano C, López $\mathbf{C}$ et al. Parásitos gastrointestinales en caninos de cinco municipios del Huila y su importancia en salud pública. Infectio 2004;8:138.

9. Hill's Pet Nutrition, Inc. Pet body condition scoring/ weight. (En línea) 1999 Jun-Jul [consultado 2004 sep 3]. Disponible en: http://216.81.250.195/public/nutrition/ bcs.2.asp.

10. Laboratorio de Parasitología Veterinaria. Protocolos del Laboratorio de Parasitología, Facultad de Medicina Veterinaria y de Zootecnia. Santafé de Bogotá: Universidad Nacional de Colombia; 2003. p.42.

11. Rep BH. Intestinal helminthes in dogs and cats on the Antillian islands Aruba, Curacao and Bonaire. Trop Geogr Med 1975;27:317-23.

12. Hassan IC. Gastrointestinal helminth parasites of dogs in the Western Area-Freetown (Sierra Leone). Beitr Trop Landwirtsch Veterinarmed 1982;20:401-7.

13. Ugochukwu EI, Ejimadu KN. Comparative studies on the infestation of three different breeds of dogs by gastro-intestinal helminths. Int J Zoonoses 1985;12: 318-22.

14. Ugochukwu EI, Ejimadu KN. Studies on the prevalence of gastro-intestinal helminths of dogs in Calabar, Nigeria. Int J Zoonoses 1985;12:214-8.

15. Ulon MG, Bottinelli SN, Meza OR, Lotero ZD,Ruiz DA, Raquel M. Determinación parasitaria en materia fecal de perros y gatos de la ciudad de Corrientes, Argentina. Universidad Nacional del Nordeste. Comunicaciones Científicas y Tecnológicas 2000. Disponible: http://www.unne.edu.ar/cyt/2000/4_ veterinarias/v_pdf/v_001.pdf

16. Segovia T, Ozuna R. Aspectos clínicos, terapéuticos y zoonóticos en las infestaciones gastrointestinales. Revista de Ciencia y Tecnología, Dirección de Investigaciones, Universidad Nacional Asunción, Paraguay 2000;1:97.

17. Minnaar WN, Krecek RC. Helminths in dogs belonging to people in a resource-limited urban community in Gauteng, South Africa. Onderstepoort J Vet Res 2001;68:111-7. Disponible en: http://www. findarticles. com/p/articles/mi_qa3910/is_200106/ai_n9003342

18. Byron L, Blagburn MS. Prevalence of canine and feline parasites in the United States. Compend Contin Educ Pract Vet 2001;23(Suppl.):1.

19. Fernández CF, Cantón AG. Frecuencia de helmintos en intestino de perros sin dueño sacrificados en la ciudad de Querétaro, México. Vet Mex 2002;33:247.

20. Acha PN, Syfres B. Zoonosis y enfermedades transmisibles comunes al hombre y a los animales. $2^{\mathrm{a}}$ ed. Washington, D. C.: Organización Panamericana de la Salud/Organización Mundial de la Salud; 1986. p.727. 
21. Botero D, Restrepo M. Parasitosis humanas. $4^{\mathrm{a}}$ edición. Medellín, Colombia: Corporación para Investigaciones Biológicas; 2003. p.93.

22. Cardona G. El clima y el ciclo biológico del parasitismo gastrointestinal. e-campo (en línea) 2004 Sept-Oct [Consultado 2004 Oct 1]. En http://www.e-campo.com/ sections/news/display.php/uuid.441ED0D0-6535486F-85A27CFA2EBB8408/catUuid.91D0E3D4-E26911D3-A5140006292E2740/2004.

23. Cordero del Campillo M, Rojo VFA, Martínez FAR, Sánchez AMC, Hernández RS, Navarrete LCl et al. Parasitología veterinaria. Madrid, España: McGraw-Hill; 2000.

24. Alarcón V, Bautista J. Efectividad del febendazol en suspensión al $10 \%$ en helmintiasis caninas en Bogotá. (tesis). Santa fe de Bogotá: Universidad de Ciencias Aplicadas y Ambientales; 1989.

25. Lightner L, Christensen B, Beran G. Epidemiologic findings on canine and feline intestinal nematode infections from records of the lowa State University Veterinary Clinic. J Am Vet Med Assoc 1978;172: 564-7.

26. Gaxiola CS, Obregón JF, Domínguez JE, Pérez CJ, Caro PJ, Martínez GM et al. Frecuencia de parásitos gastrointestinales en perros de Culiacán, Sinaloa, México. Revista, XII Congreso Nacional de Parasitología (México) 1996;67.

27. Iannacone J, Córdova K, Wong R. Estructura comunitaria de helmintos enteroparásitos en perros vagabundos de San Juan de Lurigancho, Lima, Perú. (tesis). Lima: Universidad Nacional Mayor de San Marcos; 1999.

28. Fok E, Szatmari V, Busak K, Rozgonyi F. Prevalence of intestinal parasites in dogs in urban and rural areas of Hungary. Vet Q 2001;23:96-8.

29. Rodríguez RI, Cob LA, Domínguez-Alpizar JL. Frecuencia de parásitos gastrointestinales en animales domésticos diagnosticados en Yucatán, México. Revista Biomed 2001;12:19.

30. Trillo-Altamirano MP, Carrasco AJ, Cabrera R. Prevalencia de helmintos enteroparásitos zoonóticos y factores asociados en Canis familiaris en una zona urbana de la ciudad de Ica, Perú. Parasitol Latinoam 2003;58:136.

31. Agudelo C, Villareal E, Cáceres E, López C, Eljach J, Ramírez $\mathbf{N}$ et al. Human and dogs Toxocara canis infection in a poor neighborhood in Bogotá. Mem Ins Oswaldo Cruz 1990;85:75-8.

32. El-Shehabi FS, Abdel-Hafez SK, Kamhawi SA. Prevalence of intestinal helminths of dogs and foxes from Jordan. Parasitol Res 1999;85:928-34.

33. Díaz J, Chávez A, Casas E. Comparación de dos métodos convencionales de diagnostico de nematodos intestinales en Canis familiaris con el examen post mortem. Rev Inv Vet Peru 1999;10:56.

34. Luty T, Mizgajska H. Prevalence of Toxocara spp. and other intestinal parasites in dogs and cats. Vet Med 1999;55:759. 